\title{
Heat treatment prior to testing allows detection of antigen of Dirofilaria immitis in feline serum
}

\author{
Susan E Little ${ }^{1 *}$, Melissa R Raymond ${ }^{1}$, Jennifer E Thomas' ${ }^{1}$, Jeff Gruntmeir ${ }^{1}$, Joe A Hostetler ${ }^{2}$, James H Meinkoth ${ }^{1}$ \\ and Byron L Blagburn ${ }^{3}$
}

\begin{abstract}
Background: Diagnosis of Dirofilaria immitis infection in cats is complicated by the difficulty associated with reliable detection of antigen in feline blood and serum samples.

Methods: To determine if antigen-antibody complex formation may interfere with detection of antigen in feline samples, we evaluated the performance of four different commercially available heartworm tests using serum samples from six cats experimentally infected with $D$. immitis and confirmed to harbor a low number of adult worms (mean =2.0). Sera collected $168(n=6), 196(n=6)$, and $224(n=6)$ days post infection were tested both directly and following heat treatment.

Results: Antigen was detected in serum samples from 0 or 1 of 6 infected cats using the assays according to manufacturer's directions, but after heat treatment of serum samples, as many as 5 of 6 cats had detectable antigen 6-8 months post infection. Antibodies to $D$. immitis were detected in all six infected cats by commercial in-clinic assay and at a reference laboratory.

Conclusions: These results indicate that heat treatment of samples prior to testing can improve the sensitivity of antigen assays in feline patients, supporting more accurate diagnosis of this infection in cats. Surveys conducted by antigen testing without prior heat treatment of samples likely underestimate the true prevalence of infection in cats.
\end{abstract}

Keywords: Antigen test, Cat, Dirofilaria immitis, Heartworm, Heat treatment

\section{Background}

Detection of antigen of Dirofilaria immitis is a sensitive and specific means of diagnosing heartworm infection in dogs, but these tests have been considered much less reliable in cats [1-3]. Lack of antigen detection in cats has been attributed to low circulating antigenemia due to the low number of worms often seen in feline infections, a higher likelihood of male only infections, and stunted development of $D$. immitis adults in an aberrant host [4-6]. In dogs, these assays are considered capable of detecting infections with as few as $1-3$ adult female D. immitis $[1,7]$.

When antigen-based assays were first developed for detecting $D$. immitis in dogs, serum was pre-treated with

\footnotetext{
*Correspondence: susan.little@okstate.edu

${ }^{1}$ Department of Veterinary Pathobiology, Center for Veterinary Health

Sciences, Oklahoma State University, Stillwater, OK 74078, USA

Full list of author information is available at the end of the article
}

heat and/or EDTA to destroy immune complexes prior to testing, as antigen-antibody complexes were recognized as inhibiting detection of antigen in some canine samples [8-10]. However, this step is no longer included in protocols of commercial $D$. immitis antigen tests, including those labeled for use in cats. We recently described that heat treatment of canine serum samples prior to testing can reveal antigen of $D$. immitis, a phenomenon which may interfere with detection of infection in as many as 7\% of dogs in the southern United States [S. Little, unpublished data]. In canine serum samples which test false negative on commercial assays, antigen is apparently trapped in immune complexes, preventing detection. Once these complexes are disrupted, the assays are able to accurately detect antigen [8-10].

To determine the role antigen blocking may play in detection of $D$. immitis antigen in feline samples, we tested serum samples from cats experimentally infected with a 
low number of heartworms with four different commercially available assays before and after heat treatment of sera.

\section{Methods Samples}

Large volume serum samples were available from six (3 male and 3 female) domestic short-haired cats experimentally infected at 10 months of age with Dirofilaria immitis by subcutaneous inoculation of third-stage larvae $\left(\mathrm{L}_{3}\right)$ and confirmed to be infected by recovery of adult worms at necropsy or confirmation of histological lesions. Briefly, trickle infection of a total of $100 \mathrm{~L}_{3}$ of $D$. immitis was performed by subcutaneous inguinal inoculation of each cat a total of four times, on study days 7, 14, 21, and 28 , with $25 \mathrm{~L}_{3}$ (Missouri strain) harvested shortly prior to inoculation from infected Aedes aegypti mosquitoes (Liverpool strain). Whole blood samples were collected from the jugular vein or, rarely, the cephalic vein, of each cat on days $84,112,140,168,196$, and 224 directly into vacuum tubes containing either EDTA or no additive. Cats were cared for through Oklahoma State University's Association for Assessment and Accreditation of Laboratory Animal Care-accredited animal resources program throughout the study; all research procedures followed a detailed animal care and use protocol approved by Oklahoma State University's Institutional Animal Care and Use Committee. Anti-coagulated whole blood was assayed for microfilaria by modified Knott's test and by real-time PCR for Wolbachia spp. as previously described $[11,12]$. For tubes without additive, blood was allowed to clot, the serum separated by centrifugation, placed into aliquots, and stored at $-80^{\circ} \mathrm{C}$ until further use.

\section{Antibody testing}

Antibody testing was conducted using a commercial assay (Solo Step ${ }^{\circ}$ FH, HESKA) according to manufacturer's instructions, and by a fee-for-service reference laboratory (ANTECH Diagnostics) using a commercially available microtiter plate assay (Synbiotics Corporation, Zoetis). The reference laboratory tested each sample in triplicate and provided optical density (O.D.) results from each well as determined on spectrophotometry as well as corresponding positive and negative control sample wells.

\section{Antigen testing}

All antigen testing was conducted using commercial assays according to manufacturers' instructions, with the exception that samples were tested before and after heat treatment on each assay. For heat treatment, serum samples were placed in a heat block at $103^{\circ} \mathrm{C}$ for 10 minutes, the resultant coagulum centrifuged, and the supernatant used in each commercial assay. Test kits evaluated before and after heat treatment included enzyme linked immunosorbent assay (ELISA) in microtiter plate formats (DiroCHEK ${ }^{\circ}$, Synbiotics Corporation, Zoetis; PetChek ${ }^{\circ}$ Heartworm PF Antigen Test, IDEXX Laboratories, Inc.), membrane bound ELISAs (SNAP ${ }^{\circ}$ Feline Heartworm ${ }^{\bullet}$ Test, IDEXX Laboratories, Inc.), and lateral flow immunochromatographic tests (WITNESS ${ }^{\circ}$ HW, Synbiotics Corporation, Zoetis). In addition, O.D. readings were obtained by spectrophotometry before and after heat treatment for one of the microtiter plate assays (PetChek ${ }^{\circ}$ Heartworm PF Antigen Test, IDEXX Laboratories, Inc.) according to manufacturer's directions.

\section{Comparison of test results}

Optical density from microtiter plate assay (PetChek ${ }^{\circ}$ Heartworm PF Antigen Test, IDEXX Laboratories, Inc.) and mean O.D. of triplicate antibody tests (ANTECH Diagnostics) for each cat for all days on which antigen was detected in any one cat and for cats on each of three individual study days (study days 168, 196, and 224) were compared using one-way ANOVA in Excel 2007 (Microsoft Office) with significance assigned at alpha = 0.05 [13]. An antigen/antibody ratio was also determined for each cat on days 168, 196, and 224 using O. D. and mean O. D., respectively.

\section{Results}

All six cats developed infection with $D$. immitis as evidenced by detection of classic lesions on histopathologic examination or recovery of adult worms at necropsy. A total of $1-6$ adult $D$. immitis were collected from 5 cats; adult worms were not recovered from the remaining cat at necropsy but severe pulmonary lesions consistent with D. immitis infection were present on histopathologic examination (Table 1). Microfilariae of $D$. immitis were not detected on any day by Knott's test of whole blood, and real time PCR for Wolbachia spp. on whole blood was consistently negative. Antibodies to $D$. immitis were detected by both assays in five cats on day 84 , and in all six cats on study days 112, 140, 168, 196, and 224. Antigen of $D$. immitis was detected in 0,1 , and 1 cat on days 168,196 , and 224 , respectively, when testing was performed according to manufacturer's instructions, without prior heating, using the microtiter well assays. When testing was performed with membrane bound ELISA and lateral flow immunochromatographic tests, antigen was detected in 0 or 1 cat, respectively.

After heat treatment of samples, the microtiter well assays identified antigen in 1, 5, and 5 of 6 cats on days 168,196 , and 224, respectively. Antigen was also detected in 4 of 6 cats after heating samples collected on day 224 using both the membrane bound ELISA and the lateral flow immunochromatographic test. Results (O.D.) on one of the microtiter plate assays (PetChek Heartworm PF Antigen Test, IDEXX Laboratories, Inc.) before and after 
Table 1 Results from testing feline serum samples collected 196 and 224 days after experimental infection with 100 third-stage larvae of Dirofilaria immitis (positive results in bold)

\begin{tabular}{|c|c|c|c|c|c|c|c|c|}
\hline \multirow[b]{2}{*}{$\begin{array}{c}\text { Cat (number of } \\
\text { worms recovered) }\end{array}$} & \multicolumn{4}{|c|}{ Day 196} & \multicolumn{4}{|c|}{ Day 224} \\
\hline & $\begin{array}{l}\text { Antigen before } \\
\text { heating (O. D.) }\end{array}$ & $\begin{array}{c}\text { Antigen after } \\
\text { heating (O. D.) }\end{array}$ & Antibody & $\begin{array}{c}\text { Antigen/ } \\
\text { antibody ratio }\end{array}$ & $\begin{array}{l}\text { Antigen before } \\
\text { heating (O. D.) }\end{array}$ & $\begin{array}{l}\text { Antigen after } \\
\text { heating (O. D.) }\end{array}$ & Antibody & $\begin{array}{c}\text { Antigen/ } \\
\text { antibody ratio }\end{array}$ \\
\hline $1(0)^{*}$ & 0.157 & 0.666 & 0.529 & 1.259 & 0.059 & 0.604 & 0.396 & 1.525 \\
\hline $2(6)$ & 0.070 & 0.587 & 1.301 & 0.451 & $0.099^{* *}$ & 1.985 & 1.605 & 1.237 \\
\hline $3(1)$ & 0.064 & 0.289 & 0.264 & 1.095 & 0.056 & $0.120^{* *}$ & 0.509 & 0.236 \\
\hline $4(2)$ & 0.055 & 0.081 & 0.578 & 0.140 & 0.060 & 0.068 & 0.528 & 0.129 \\
\hline $5(1)$ & 0.060 & 0.146 & 1.111 & 0.131 & 0.074 & 1.921 & 1.329 & 1.445 \\
\hline $6(2)$ & 0.050 & 0.191 & 0.374 & 0.511 & 0.057 & 0.579 & 1.387 & 0.417 \\
\hline Mean (2.0) & 0.076 & 0.327 & 0.693 & 0.598 & 0.068 & 0.880 & 0.959 & 0.832 \\
\hline Number positive & $1 / 6$ & $5 / 6$ & $6 / 6$ & & $1 / 6$ & $5 / 6$ & $6 / 6$ & \\
\hline
\end{tabular}

*No adult worms recovered but severe pulmonary lesions identified on histologic examination of lung sections. ${ }^{* *}$ Antigen was detected by visual color change but did not exceed the manufacturer's specified cutoff (negative control +0.05 ) for positive on the spectrophotometric assay used (PetChek ${ }^{\circledR}$ Heartworm PF Antigen Test, IDEXX Laboratories, Inc).

Positive results in bold.

heating, and of antibody assay from the same samples, for days 196 and 224 are shown in Table 1. Results (O.D.) of antigen test (PetChek ${ }^{\odot}$ Heartworm PF Antigen Test, IDEXX Laboratories, Inc.) after heating and antibody tests were significantly associated when all days were considered together $\left(R^{2}=0.40, F=16.6, P<0.001\right)$ and on samples from day $224\left(R^{2}=0.70, F=16.1, P=0.005\right)$ but not for days $168(F=2.6, P=0.15)$ or $196(F=0.36$, $P=0.56)$ alone. The antigen/antibody ratio was highest in the single cat for which antigen was detected prior to heating on day 196 (Table 1), although the limited number of samples precluded meaningful statistical analysis.

\section{Discussion}

In the present study, we showed that heat treatment of feline serum prior to antigen testing resulted in a dramatic increase in detection of $D$. immitis antigen. Although only $1 / 6$ (16.7\%) samples from cats infected with a low number of adult heartworms was antigen positive prior to heat treatment, as many as 5/6 (83.3\%) became positive after heat treatment when the most sensitive microtiter well plate assays were used, presumably due to destruction of antibody and release of antigen from antibody-antigen complexes [8]. Accordingly, antigen blocking resulted in a false negative result from the majority $(80 \%)$ of cats harboring both adult $D$. immitis and circulating antigen. Given the findings in the present study, it seems prudent to heat treat serum samples from cats with a high index of suspicion for heartworm infection prior to testing for antigen of $D$. immitis.

Although the American Heartworm Society feline guidelines specifically state that "antigen tests are more reliable [in cats] than generally credited," many still have the impression that because cats generally have very low worm burdens or male-only worm infections, antigen tests are not useful in cats $[4,6]$. The data in the present study are exciting in that they suggest that once antigenantibody complexes are disrupted, antigen tests may be of great value in confirming feline infection with D. immitis, particularly the microtiter well-based assays. Heat treatment of samples prior to testing may have clinical utility, particularly given the low level of circulating microfilariae usually present in cats and difficulties in interpreting antibody test results [2,5]. This finding may be of use not only in confirming an initial clinical suspicion of feline heartworm infection, but also for evaluating the phenomenon of self-cure in cats, which previously required evaluation of antibody levels 12 months after antigen of $D$. immitis was no longer identified [14]. The prevalence of $D$. immitis antigen in feline samples in the United States is estimated to be $0.9 \%$ [15], but the data in the present study suggest that the actual prevalence is likely higher, particularly in areas where canine heartworm infection and thus spillover into cats, is endemic or hyperendemic.

Interestingly, heat treatment of sera allowed us to detect antigen in almost every infected cat included in the present study. The basis for interference with antigen detection is not fully understood, but in other systems this process is thought to be related to antigen-antibody complex formation $[16,17]$. Cats infected with $D$. immitis develop a remarkable degree of inflammation which manifests as severe lung pathology characterized by villous arteritis, medial hypertrophy of the pulmonary arterioles, and pulmonary parenchymal damage, collectively referred to as heartworm-associated respiratory disease (HARD) [2,3,5]. This chronic inflammatory process likely results in hypergammaglobulinemia, which may prevent detection of $D$. immitis antigen by either specific or non-specific formation of antigen-antibody complexes in vivo $[8,18]$. Elucidation of the mechanisms responsible for inhibition of antigen detection in feline samples as well as the 
best approach to disrupt these complexes will require additional research.

\section{Conclusions}

Antigen tests for D. immitis have long been considered of limited utility in cats. The data in the present study suggest this concept should be revisited. Heating feline serum samples prior to testing greatly facilitated detection of $D$. immitis antigen, presumably due to disruption of antigen-antibody complexes.

\section{Competing interests}

In the past five years, SL, JT, and BB have received reimbursement, speaking fees, or research support from IDEXX Laboratories and Pfizer Animal Health (now Zoetis), manufacturers of D. immitis antigen tests, or from Bayer Animal Health, a company that manufactures heartworm preventives, including those for cats. In addition, $\mathrm{JH}$ is an employee of Bayer Animal Health. The other authors $(M R, J G, J M)$ have no competing financial interests to disclose.

\section{Authors' contributions}

$\mathrm{SL}, \mathrm{BB}$, and JM conceived of the study, SL and JH participated in its design and execution and drafted the manuscript, and MR and JG carried out the antigen and antibody testing. JT led the completion of the study, particularly the experimental infections. All authors read and approved the final version of the manuscript.

\section{Acknowledgements}

Funding to support the feline experimental infections which provided the samples used in this study was provided by Bayer HealthCare, Animal Health. Additional funding was provided by the Krull-Ewing Endowment at Oklahoma State University and the National Center for Veterinary Parasitology, which receives annual support from Bayer HealthCare, Animal Health, Novartis Animal Health, and Merial, Limited, a Sanofi Company. Dr. Lindsay Starkey, Anne Barrett, Kaylynn Gruntmeir, and the Laboratory Animal Resources staff at the Center for Veterinary Health Sciences provided outstanding technical assistance.

\section{Author details}

${ }^{1}$ Department of Veterinary Pathobiology, Center for Veterinary Health Sciences, Oklahoma State University, Stillwater, OK 74078, USA. ${ }^{2}$ Bayer HealthCare, Animal Health, Shawnee Mission, Shawnee, KS, USA. ${ }^{3}$ Department of Veterinary Pathobiology, College of Veterinary Medicine, Auburn University, Auburn, AL, USA.

Received: 2 November 2013 Accepted: 17 December 2013 Published: 13 January 2014

\section{References}

1. Atkins CE: Comparison of results of three commercial heartworm antigen test kits in dogs with low heartworm burdens. J Am Vet Med Assoc 2003, 222:1221-1223.

2. McCall JW, Genchi C, Kramer LH, Guerrero J, Venco L: Heartworm disease in animals and humans. Adv Parasitol 2008, 66:193-285.

3. Lee AC, Atkins CE: Understanding feline heartworm infection: disease, diagnosis, and treatment. Top Companion Anim Med 2010, 25:224-230.

4. Hoch H, Strickland K: Canine and feline dirofilariasis: life cycle, pathophysiology, and diagnosis. Compend Contin Educ Pract Vet 2008, 30:133-141.

5. Nelson CT: Feline heartworm infection: diagnosis and management. Compend Contin Educ Pract Vet 2008, 30:393-400.

6. Graham W, Rubin SB, Boeckh A, Burhardt LF, Jones S, Miller M, Payne $P$, Rehm C, Smith-Blackmore M, Stannard R, Nelson CT, Atkins C, Carithers D, McCall J, Von Simson C: Current canine guidelines for the diagnosis, prevention, and management of heartworm (Dirofilaria immitis) infection in cats (revised January 2012). www.heartwormsociety.org, accessed October 1, 2013

7. Courtney $\mathrm{CH}$, Zheng Q-Y: Comparison of heartworm antigen test kit performance in dogs having low heartworm burdens. Vet Parasitol 2001, 96:317-322
8. Weil GJ, Malane MS, Powers KG, Blair LS: Monoclonal antibodies to parasite antigens found in the serum of Dirofilaria immitis-infected dogs. J Immunol 1985, 134:1185-1191.

9. Brunner $\mathrm{CJ}$, Hendrix CM, Blagburn BL, Hanrahan LA: Comparison of serologic tests for detection of antigen in canine heartworm infections. J Am Vet Med Assoc 1988, 192:1423-1427.

10. Tonelli QJ, Quentin AB: Factors affecting the accuracy of enzyme immunoassays for Dirofilaria immitis adult antigen. In Proceedings Am Heartworm Symp. Washington, DC: American Heartworm Society; 1989:161-165.

11. Zajac AM, Conboy GA: Detection of parasites in the blood. In Veterinary Clinical Parasitology. 8th edition. West Sussex: John Wiley and Sons, Inc; 2012:185-211

12. Turba ME, Zambon E, Zannoni A, Russo S, Gentilini F: Detection of Wolbachia DNA in blood for diagnosing filaria-associated syndromes in cats. J Clin Microbiol 2012, 50:2624-30.

13. Cross CL, Daniel WW: Biostatistics: a foundation for analysis in the health sciences. 10th edition. John Wiley and Sons, Inc: West Sussex; 2013.

14. Venco L, Genchi C, Genchi M, Grandi G, Kramer LH: Clinical evolution and radiographic findings of feline heartworm infection in asymptomatic cats. Vet Parasitol 2008, 158:232-7.

15. Lorentzen L, Caola AE: Incidence of positive heartworm antibody and antigen tests at IDEXX Laboratories: trends and potential impact on feline heartworm awareness and prevention. Vet Parasitol 2008, 158:183-90.

16. Wheat $\mathrm{L}$, Walsh $\mathrm{TJ}$ : Diagnosis of invasive aspergillosis by galactomannan antigenemia detection using an enzyme immunoassay. Eur J Clin Microbiol Infect Dis 2008, 27:245-251.

17. Swartzentruber S, LeMonte A, Witt J, Fuller D, Davis T, Hage C, Connolly P, Durkin $\mathrm{M}$, Wheat $\mathrm{L}$ : Improved detection of Histoplasma antigenemia following dissociation of immune complexes. Clin Vacc Immunol 2009, 16:320-322

18. Weil GJ, Malane MS, Powers KG: Detection of circulating parasite antigens in canine dirofilariasis by counterimmunoelectrophoresis. Am J Trop Med Hyg 1984, 33:425-430.

doi:10.1186/1756-3305-7-1

Cite this article as: Little et al: Heat treatment prior to testing allows detection of antigen of Dirofilaria immitis in feline serum. Parasites \& Vectors 2014 7:1.

\section{Submit your next manuscript to BioMed Central and take full advantage of:}

- Convenient online submission

- Thorough peer review

- No space constraints or color figure charges

- Immediate publication on acceptance

- Inclusion in PubMed, CAS, Scopus and Google Scholar

- Research which is freely available for redistribution 